\title{
Les Arméniens, les Grecs et les Juifs originaires de Grèce et de Turquie, à Paris de 1920 à 1936
}

\section{Michel Garin}

\section{Q OpenEdition}

\section{Journals}

Édition électronique

URL : https://journals.openedition.org/ceb/837

DOI : $10.4000 /$ ceb.837

ISSN : 2261-4184

Éditeur

INALCO

\section{Édition imprimée}

Date de publication : 30 mars 2011

Pagination : 367-378

ISBN : 978-2-85831-189-7

ISSN : 0290-7402

\section{Référence électronique}

Michel Garin, «Les Arméniens, les Grecs et les Juifs originaires de Grèce et de Turquie, à Paris de 1920 à 1936 », Cahiers balkaniques [En ligne], 38-39 | 2011, mis en ligne le 06 décembre 2011, consulté le 06 juillet 2021. URL : http://journals.openedition.org/ceb/837 ; DOI : https://doi.org/10.4000/ceb. 837

Ce document a été généré automatiquement le 6 juillet 2021.

\section{(c) (7) \&)}

Cahiers balkaniques est mis à disposition selon les termes de la Licence Creative Commons Attribution - Pas d'Utilisation Commerciale 4.0 International. 


\title{
Les Arméniens, les Grecs et les Juifs originaires de Grèce et de Turquie, à Paris de 1920 à 1936
}

\author{
Michel Garin
}

1 Après la Première Guerre mondiale, les autorités françaises organisent une immigration massive, mais sélective quant à l'origine géographique des immigrés, en contractant des accords sur le séjour des travailleurs étrangers avec différents pays d'émigration comme la Pologne, l'Italie, l'Espagne, la Belgique, l'Autriche. Parallèlement à cette immigration organisée, se développe une immigration spontanée qui n'est que tolérée par les autorités. C'est le cas de l'immigration originaire de Grèce et de Turquie. Les listes nominatives des recensements de 1926, 1931 et 1936 pour Paris, et les immatriculations au registre du commerce de la Seine entre 1920 et 1936 permettent de reconstituer le parcours familial, résidentiel et professionnel de plus de 19000 immigrés nés en Grèce ou en Turquie. En croisant l'origine communautaire et l'origine géographique de ces immigrés, on peut distinguer cinq groupes: les Arméniens de Turquie, les Grecs de Grèce, les Grecs de Turquie, les Juifs de Grèce et les Juifs de Turquie. À Paris, en 1926, les Grecs de Grèce sont plus nombreux que les Juifs de Grèce, mais c'est l'inverse en 1936. Chez les immigrés originaires de Turquie, la hiérarchie des effectifs reste la même tout au long de la période 1926-1936 : les Juifs sont les plus nombreux que les Arméniens, eux-mêmes plus nombreux que les Grecs. différentes.

Les Arméniens de Turquie sont les survivants des massacres et des déportations, dont leur communauté a été victime de la part du pouvoir ottoman en 1915 et dans les années postérieures. Ils sont victimes aussi, à partir de décembre 1920, de l'empêchement de toute vie communautaire dans les provinces arméniennes de Turquie, attribuées à l'Arménie par le traité de Sèvres d'août 1920, mais retombées sous domination turque, en décembre 1920. L'émigration des Arméniens de Turquie est définitive, car elle entraîne leur bannissement du territoire turc. 
4 Après la défaite militaire des Grecs dans leur guerre contre les Turcs, en 1922, les Grecs de Turquie sont expulsés de Turquie vers la Grèce au terme de l'accord d'échange des populations grecque et turque de janvier 1923, accord confirmé par le Traité de Lausanne de juillet 1923. Mais la Grèce ne peut donner du travail à l'ensemble de cette énorme population de réfugiés, un nombre important d'entre eux vont donc émigrer. Dans la continuité de l'émigration de 1916-1917, organisée par les autorités françaises pour l'industrie de guerre, un certain nombre de Grecs originaires de Turquie partent vers la France après la Première Guerre mondiale. Les Grecs de Constantinople qui eux n'entrent pas dans le cadre de l'échange obligatoire des populations doivent cependant renoncer, après l'avènement de la République turque en 1923, à leurs droits spécifiques reconnus par le Traité de Lausanne. Certains choisissent aussi d'émigrer en France.

5 Les Juifs de Grèce, dont la plus grande partie réside à Salonique, sont devenus citoyens grecs depuis le rattachement de la Macédoine à la Grèce, en 1913, après la Seconde Guerre balkanique. Ils sont les principales victimes, en 1917, de l'incendie de Salonique qui ravage presque totalement les quartiers juifs de la ville. La reconstruction de la ville s'accompagne d'une redistribution négociable des droits fonciers, qui se révèle préjudiciable aux Juifs. En outre, dans les années 1920, les Juifs de Salonique supportent mal la politique d'hellénisation de leur communauté menée par les autorités grecques, avec des mesures comme l'instauration du repos dominical, la conscription obligatoire et l'obligation pour tous les enfants de nationalité grecque de fréquenter des écoles publiques grecques. Pour ces raisons et à cause aussi de l'antisémitisme croissant, beaucoup de Juifs de Salonique choisissent d'émigrer vers les capitales européennes, dont Paris, venant ainsi grossir la petite communauté séfarade qui existait déjà avant la guerre.

6 Comme les Arméniens de Turquie et les Grecs de Constantinople, les Juifs de Turquie sont victimes, à partir de 1923, de la remise en cause par la République turque de leurs droits spécifiques reconnus par le traité de Lausanne ainsi que d'une discrimination de fait dans l'accès à certains emplois. Un nombre important de Juifs de Turquie émigrent vers Paris.

7 Parmi les cinq groupes, seuls les Grecs de Grèce émigrent pour des raisons purement économiques. L'émigration massive des Grecs, qui commence dans le Péloponnèse en 1890 suite à la crise de l'exportation du raisin noir, se dirige au départ presque exclusivement vers les États-Unis. Mais, à partir de 1916, elle se tourne aussi vers la France, en raison de la difficulté de traverser l'Atlantique du fait de la guerre sousmarine, de la campagne, brève, mais intense, de recrutement de travailleurs grecs par les autorités françaises en 1916 et 1917 et enfin de la limitation par les autorités américaines, à partir de 1917, de l'immigration sur le territoire des États-Unis. Après la Première Guerre mondiale, un certain nombre de Grecs de Grèce se dirigent donc vers la France plutôt que vers l'Amérique.

8 L'intégration professionnelle est différenciée suivant les groupes, aussi bien pour ce qui concerne le taux d'activité que pour la répartition des activités professionnelles. Les Grecs et les Arméniens ont un taux d'activité plus élevé que celui des Juifs, ils se tournent majoritairement vers l'artisanat et l'industrie alors que les Juifs se tournent surtout vers le commerce.

9 Le mariage, qu'il soit mixte ou non, est un facteur d'intégration puisqu'il ancre le parcours d'immigration dans la durée. Mais le comportement matrimonial des 
immigrés diffère statistiquement d'un groupe à l'autre, le taux de mixité des mariages étant plus fort chez les Grecs que chez les Arméniens et que chez les Juifs.

10 L'intégration dans la société française est au départ largement facilitée par la structuration forte de la vie communautaire en associations diverses, qu'il s'agisse de la pratique religieuse, de la solidarité entre les membres de la communauté, de l'encadrement de la jeunesse, ou du développement des activités culturelles et sportives.

11 L'intégration passe aussi par la participation à la guerre dans l'armée française. Dans chacun des groupes, des immigrés ont combattu aux côtés de la France au cours de la Première Guerre mondiale et certains sont devenus membres, par la suite, d'associations communautaires d'anciens combattants.

Un autre signe d'intégration des communautés est l'activité intellectuelle et artistique en France de certains de leurs membres. Bien avant 1920, des intellectuels arméniens et grecs ont fait des séjours à Paris où s'y sont installés. Entre 1920 et 1936, l'activité intellectuelle de ces communautés est plus intense que celle de la communauté juive originaire de Grèce et de Turquie où une élite intellectuelle n'apparaît qu'à la fin des années 1930.

13 L'intégration progressive des immigrés dans la société française n'empêche pas le maintien de l'intérêt qu'ils portent au devenir de leur nation d'origine ou de référence. Les Arméniens de Turquie résidant à Paris s'intéressent, au sein d'organisations politiques localement bien structurées, au devenir de la République arménienne, soviétisée dès 1920. Les Grecs de Paris s'intéressent eux, au travers de leur presse, à la politique intérieure grecque et à l'évolution de la situation politique en Grèce. De plus, un certain nombre d'hommes politiques et de militants grecs se réfugient à Paris, avant et après l'instauration en 1936 de la dictature en Grèce. En revanche, avant la Deuxième Guerre mondiale, les Juifs originaires de Grèce et de Turquie ne s'impliquent qu'assez peu, à Paris, dans le mouvement sioniste.

Dans la période 1920-1936, en France, l'accès des étrangers à l'activité salariée est très étroitement réglementé, notamment à travers la carte d'identité de travailleur étranger, avec des conditions de plus en plus restrictives de durée de validité et de secteur d'emploi autorisé. De plus les étrangers sont victimes, plus que les Français, de la crise économique, les autorités réagissant à cette crise, à partir de 1932, par l'application de quotas d'étrangers dans l'industrie. En revanche, jusqu'au milieu des années 1930 l'accès à l'activité indépendante, c'est-à-dire à l'exercice d'une profession libérale, mais surtout à l'exercice d'une activité artisanale ou commerciale, reste relativement libre pour les étrangers.

15 Dans la période 1920-1936, au sein de l'immigration originaire de Grèce et de Turquie l'activité indépendante se développe comme une stratégie professionnelle alternative à l'activité salariée.

16 Il existe de nombreux points communs au développement de l'activité indépendante dans les différents groupes, mais aussi des différences sensibles.

17 Le premier point commun aux différents groupes est la croissance continue, entre 1926 et 1936, du taux d'indépendance professionnelle, c'est-à-dire du rapport entre le nombre d'indépendants et le nombre d'actifs. Chez les nouveaux actifs, il y a une tendance plus marquée que chez ceux qui les ont précédés, à court-circuiter le passage par l'emploi salarié. 

l'indépendance, le taux d'essai de l'indépendance est nettement plus élevé que chez les enfants de parents actifs qui n'ont pas eu cette expérience. De plus, l'exemple de l'indépendance dans l'entourage, c'est-à-dire chez les personnes qui vivent dans le même foyer, influence fortement de façon positive le taux d'essai de l'indépendance. 
Enfin, l'exemple de l'indépendance dans le voisinage résidentiel de même origine géographique et de même originaire communautaire a également un effet positif sur l'orientation vers l'indépendance.

L'activité indépendante se développe au sein de filières professionnelles regroupant des immigrés de même origine communautaire et dans certains cas de même origine géographique. Les entreprises d'une filière se créent autour d'un ou de plusieurs pôles d'implantation géographiques. Les pôles d'implantation émergent, se développent, se saturent au fil du temps suivant un phénomène qui peut être comparé à une cristallisation. Il existe aussi un phénomène d'émulation des groupes communautaires, les immigrés d'un groupe créant parfois, avec un certain décalage, des entreprises dans la même filière et le même pôle d'implantation qu'une ou plusieurs autres groupes antérieurement implantés dans ce pôle.

Les indépendants qui coopèrent en conservant leur statut d'entrepreneur individuel le font, très généralement, avec des immigrés de même origine communautaire, souvent de surcroît de même pays de naissance et, assez souvent, de même ville de naissance. Chez les indépendants associés au sein de sociétés, on observe dans les cinq groupes une fréquence très forte d'association avec des indépendants de même pays de naissance et de même origine communautaire.

Si l'activité indépendante des cinq groupes d'immigrés originaires de Grèce et de Turquie a de nombreux points communs, elle présente aussi des différences très sensibles. En premier lieu, il y a des différences sensibles de taux d'indépendance professionnelle entre les différents groupes. La hiérarchie des valeurs du taux d'indépendance reste la même tout au long de la période 1926-1936. Le taux d'indépendance le plus élevé est celui des Juifs de Grèce devant celui des Juifs de Turquie, celui des Grecs de Grèce, celui des Grecs de Turquie et celui des Arméniens de Turquie.

29 Le taux d'indépendance, à l'intérieur d'une communauté, dépend du pays naissance, mais cette dépendance est moindre que celle de l'origine communautaire. Ainsi, les Juifs de Turquie sont relativement moins souvent indépendants que les Juifs de Grèce, mais ils sont relativement plus souvent indépendants que les Grecs et les Arméniens. Les Grecs de Turquie sont moins souvent indépendants que les Grecs de Grèce, mais plus souvent indépendants que les Arméniens de Turquie.

30 La hiérarchie des taux de premières créations immatriculées au registre du commerce par rapport à l'effectif des salariés est un peu différente. Si le taux de premières créations chez les Juifs de Grèce et des Juifs de Turquie est supérieur à celui des autres groupes, on observe que, chez les Grecs de Turquie et les Arméniens de Turquie, le taux est supérieur à celui que l'on observe chez Grecs de Grèce. Cette différence s'explique notamment par le fait que, suite à un licenciement, les Grecs de Grèce ont la possibilité de retourner au pays, ce qui n'est pas le cas pour les immigrés des autres groupes. Cependant, les différences de valeurs des taux d'indépendance entre les groupes s'atténuent au long de la période 1926-1935.

31 Les Juifs ont tendance, plus souvent que les Arméniens et les Grecs, à choisir l'indépendance à leur arrivée à Paris et, pour ceux qui sont d'abord salariés, à évoluer vers l'indépendance. Ce sont les actifs indépendants juifs de Grèce qui ont le moins souvent une étape d'activité salariée dans leur parcours professionnel. On trouve ensuite, par ordre de pourcentage croissant, les indépendants juifs de Turquie, les indépendants Grecs de Grèce, les indépendants Arméniens de Turquie et enfin les 
indépendants Grecs de Turquie. La différence de pourcentage de parcours ayant une étape salariée entre Grecs et Arméniens n'est pas importante. Les Grecs sont les plus nombreux, relativement, à avoir exercé une activité salariée dans la même profession que l'activité indépendante qu'ils créent. Il y a, chez les Grecs une phase d'apprentissage ou de formation plus fréquente que chez les autres.

L'influence de l'exemple de l'entourage et du voisinage résidentiel de mêmes origines sur l'essai de l'indépendance est beaucoup plus nette chez les Arméniens et chez les Grecs que chez les Juifs.

Les immatriculations de Juifs de Grèce dans des sociétés sont plus fréquentes que dans les autres groupes, quelle que soit la forme juridique de la société : société en nom collectif, société à responsabilité limitée ou société anonyme. C'est chez les Juifs de Grèce que l'on trouve les sociétés au capital moyen le plus important, devant celui des sociétés des Arméniens et les Grecs.

La proportion de cessations d'activité indépendante suite à décision judiciaire la plus forte est celle des Grecs de Grèce, devant celle des Grecs de Turquie, des Juifs de Turquie, des Juifs de Grèce et des Arméniens de Turquie. La politique antijuive du régime de Vichy de juillet 1940 à juillet 1944 se traduit pendant cette période, par la radiation par décision administrative de nombreuses entreprises tenues par des Juifs de Grèce et des Juifs de Turquie.

La coopération des indépendants individuels avec des immigrés de même origine communautaire et de même ville de naissance est la plus forte chez les Grecs de Castoria, les Juifs de Salonique et les Juifs de Constantinople. La coopération des indépendants associés avec des immigrés de même origine communautaire est forte dans tous les groupes, quelle que soit la ville de naissance, mais elle est presque systématique chez les Grecs de Castoria et très fréquente chez les Juifs de Salonique.

La répartition géographique des immatriculations diffère d'un groupe à l'autre. Chez les indépendants arméniens de Turquie, $50 \%$ des immatriculations sont enregistrées dans les $9^{\mathrm{e}}, 20^{\mathrm{e}}, 19^{\mathrm{e}}, 10^{\mathrm{e}}$ et $18^{\mathrm{e}}$ arrondissements, chez les indépendants Grecs de Grèce dans les $9^{\mathrm{e}}, 6^{\mathrm{e}}, 2^{\mathrm{e}}, 5^{\mathrm{e}}, 10^{\mathrm{e}}$ et $20^{\mathrm{e}}$ arrondissements, chez les indépendants Grecs de Turquie dans les $20^{\mathrm{e}}, 9^{\mathrm{e}}, 19^{\mathrm{e}}, 18^{\mathrm{e}}, 10^{\mathrm{e}}$ et $11^{\mathrm{e}}$ arrondissements, chez les indépendants juifs de Grèce dans les $9^{\mathrm{e}}, 2^{\mathrm{e}}$ et $10^{\mathrm{e}}$ arrondissements et chez les indépendants juifs de Turquie dans les $11^{\mathrm{e}}, 9^{\mathrm{e}}$ et $10^{\mathrm{e}}$ arrondissements. Les Arméniens de Turquie sont les seuls à développer de façon importante, surtout à partir de 1931, des activités indépendantes en proche banlieue parisienne, principalement à Alfortville et à Issy-les-Moulineaux. C'est la conséquence de la reconversion des salariés qui avaient été embauchés dans l'industrie et qui sont licenciés en raison de la crise économique et de la politique gouvernementale d'imposition de quotas d'étrangers.

L'activité indépendante de chacun des groupes se développe au sein de filières professionnelles propres à un groupe ou communes à plusieurs groupes. Mais la répartition des activités par filière est notablement différente dans chaque groupe.

Chez les indépendants Arméniens de Turquie, neuf filières dépassent cent immatriculations pour la période allant de juillet 1920 à mars 1936. Il s'agit, dans l'ordre d'importance, du commerce de bonneterie, tissus et confection, de la fabrication et la réparation des chaussures, du commerce de comestibles et d'épicerie, des courtiers commissionnaires représentants et voyageurs, des tailleurs et couturières, du commerce forain, de la coiffure, du commerce des objets domestiques et du commerce 
d'antiquités et de brocante. Les pôles d'implantation principaux des activités diffèrent selon les filières. Il s'agit du pôle centré sur le quartier du Mail dans le $2^{\mathrm{e}}$ arrondissement pour le commerce de la bonneterie, des tissus et de la confection, du quartier Belleville dans le $20^{\mathrm{e}}$ pour la fabrication et la réparation des chaussures, des villes d'Issy-les-Moulineaux et d'Alfortville pour l'épicerie et le commerce forain, du quartier Faubourg Montmartre dans le $9^{e}$ pour les courtiers, les tailleurs et les couturières, d'Issy-les-Moulineaux pour les coiffeurs, du quartier de la Chaussée d'Antin dans le $9^{\text {e }}$ pour le commerce des objets domestiques, les antiquaires et les brocanteurs.

Chez les indépendants Grecs de Grèce, deux filières totalisent plus de cent immatriculations pour la période allant de juillet 1920 à mars 1936: les pelletiers fourreurs et les fabricants et réparateurs de chaussures. La première se développe dans deux principaux pôles d'implantation, l'un centré sur le quartier de la Monnaie dans le $6^{e}$ et l'autre sur quartier Bonne Nouvelle dans le $2^{e}$. La plupart des pelletiers fourreurs sont originaires de la ville de Castoria située en Macédoine où cette profession existe depuis le début du XVII ${ }^{e}$ siècle. Cette filière est l'exemple d'une activité indépendante d'immigrés prolongeant une tradition professionnelle de la ville d'origine. De plus, c'est une filière dont l'implantation à Paris est antérieure à 1921. La seconde filière, celle des fabricants réparateurs de chaussures, se développe dans un pôle centré sur le quartier de Belleville. Son développement n'est significatif qu'après 1921.

Chez les indépendants grecs de Turquie, trois filières dépassent cent immatriculations au cours de la période. Il s'agit des fabricants et réparateurs de chaussures, des coiffeurs et des tailleurs et couturières. Les fabricants et réparateurs de chaussures sont principalement implantés dans un pôle centré sur Belleville, les coiffeurs dans un pôle ayant pour centre le quartier Grenelle dans le $15^{\mathrm{e}}$, les tailleurs et couturières dans un pôle centré sur Rochechouart dans le $9^{\mathrm{e}}$. Ces filières ne se développent pas de façon significative avant 1921.

Chez les indépendants juifs de Grèce, qui sont très majoritairement originaires de Salonique, trois filières professionnelles atteignent cent immatriculations sur l'ensemble de la période 1920-1936. Il s'agit des commerçants en bonneterie, tissus, confection, des courtiers commissionnaires représentants et voyageurs et des commerçants forains. Les commerçants en bonneterie, tissus et confection sont principalement implantés dans un pôle centré dans le quartier Bonne Nouvelle, les courtiers commissionnaires représentants et voyageurs dans un pôle centré sur le Faubourg Montmartre et les commerçants forains dans le quartier Roquette dans le $11^{\mathrm{e}}$. Les deux premières filières sont des filières historiques, implantées avant 1921. La filière du commerce de bonneterie, tissus, confection des immigrés juifs de Salonique prolonge une tradition professionnelle qui existait dans cette ville depuis le début du $\mathrm{XVII}$ siècle.

Chez les indépendants juifs de Turquie, dans la période antérieure à 1936, six filières professionnelles totalisent plus de cent immatriculations. Il s'agit des commerçants en bonneterie tissus et confections, des commerçants forains, des courtiers commissionnaires représentants et voyageurs, des commerçants en objets domestiques, des antiquaires et brocanteurs et des tailleurs et couturières. Les principaux pôles d'implantation des commerçants en bonneterie, tissus et confection sont Bonne et Roquette. L'implantation des forains et des tailleurs couturières est centrée sur Roquette, celle des courtiers commissionnaires représentants et voyageurs est centrée 
sur le Faubourg Montmartre dans le $9^{e}$, celle des commerçants en objets domestiques sur la Porte Saint-Denis dans le $10^{\mathrm{e}}$ et Roquette et celle des antiquaires brocanteurs sur le Faubourg Montmartre et la Madeleine dans le $8^{\mathrm{e}}$. Chez les Juifs originaires d'Andrinople, la filière du commerce de la bonneterie, tissus et confection prolonge une tradition de commerce des tissus existant dans cette ville depuis au moins le XVIII ${ }^{\mathrm{e}}$ siècle.

Les filières professionnelles, dans un groupe d'immigrés défini par l'origine communautaire et le pays de naissance, se développent soit par continuité avec une tradition professionnelle existant antérieurement dans les villes d'origine, soit par adaptation de l'activité des immigrés essayant l'indépendance, à la demande de la société d'accueil. Le groupe devient ainsi le lieu d'élaboration de nouvelles traditions professionnelles. Les indépendants juifs de Grèce et de Turquie sont à la fois plus mobiles géographiquement et plus mobiles professionnellement que les indépendants des autres groupes. À Paris, avant 1936, les organisations professionnelles sont déjà bien structurées chez les Grecs. Les organisations professionnelles n'existent que pour les professions libérales chez les Arméniens et elles sont peu développées chez les Juifs. Les associations professionnelles sont toujours propres à une communauté, mais elles peuvent regrouper des personnes originaires de Grèce et de Turquie. L'association des fourreurs grecs L'Orestias est particulière, car elle regroupe des immigrés qui sont presque tous originaires de la même ville, Castoria.

En résumé, à Paris, entre 1920 et 1936, la croissance de l'activité indépendante, son processus de développement et ses moteurs sont communs à tous les groupes d'immigrés originaires de Grèce et de Turquie. En revanche, l'intensité, l'objet, les modalités juridiques et capitalistiques et les causes de cessation de l'activité indépendante dépendent fortement de l'origine communautaire des groupes. Il y a donc, dans l'immigration, des différences socioprofessionnelles sensibles entre des groupes communautaires issus, de façon proche ou plus lointaine dans le temps, de l'espace ottoman, mais qui ont vécu dans cet espace de façon assez séparée.

\section{BIBLIOGRAPHIE}

Akgönül, Samim, (2004), Les Grecs de Turquie : processus d'extinction d'une minorité de l'âge de l'Étatnation à l'âge de la mondialisation, 1923-2001, Paris : L'Harmattan ; Louvain la Neuve : Bruylant Academia.

Benbassa, Esther, (1993), Une diaspora sépharade en transition. Istanbul XIX ${ }^{e}-X X^{e}$ siècle, Paris : Éditions du Cerf.

Bernstein, Serge, (2002), La France des années 30, Paris : Armand Colin, collection Cursus.

Bruneau, Michel, (1996), « Une immigration dans la longue durée : la diaspora grecque en France », in Espace Population Sociétés, ${ }^{\circ}$ 2-3, (p. 485-495).

Dalègre, Joëlle, (2002), Grecs et Ottomans : 1453-1923 de la chute de Constantinople à la disparition de l'Empire ottoman, Paris : L'Harmattan. 
Deslondes, Olivier, (1997), Les fourreurs de Kastoria entre la Macédoine et l'Occident, CNRS, Éditions Méditerranée.

Garin, Michel, (2010), Les Arméniens les Grecs et les Juifs originaires de Grèce et de Turquie à Paris entre 1920 et 1936, Istanbul : Les Éditions Isis, (146 p).

Garin, Michel, (2010), Les Grecs de Paris pendant la Première Guerre mondiale, Istanbul : Les Éditions Isis, (84 p).

George, Pierre, (1984), Géopolitique des minorités, Paris : PUF.

Green, Nancy L., (2002), Repenser les migrations, Paris : PUF.

Hovanessian, Martine, (1995), Les Arméniens et leurs territoires, Paris : Autrement.

Immigration, emploi chômage. Un état des lieux empirique et théorique, (1999), Rapport du CERC-

Association.

Lépidis, Clément, (1984), Des dimanches à Belleville, Charmes : ACE.

Manitakis, Nicolas, Noiriel, Gérard (dir.), (1993) L'émigration grecque en France durant la première moitié du siècle. Étude d'un phénomène migratoire à visages multiples, Paris : EHESS-ENS.

Morin, Edgar, (1989), Vidal et les siens, Paris : Seuil.

Noiriel, Gérard, (2006), Introduction à la socio-histoire, Paris : La Découverte, Collection REPÈRES.

Papadopoulou, Despina, (2004), Les Grecs à Paris à la fin du XIX ${ }^{e}$, Thèse, EHESS,

Pierron, Bernard, (1996), Juifs et chrétiens de la Grèce moderne. Histoire des relations intercommunautaires de 1821 à 1945, Paris : L'Harmattan.

Prévélakis, Georges, (2005), « Salonique entre provincialisme et cosmopolitisme », Cemoti, $\mathrm{n}^{\circ} 24$, Métropole et métropolisation.

Résultats statistiques du recensement général de la population du 7 mars 1926, (1928), Imprimerie Nationale.

Résultats statistiques du recensement général de la population du 8 mars 1931, (1933), Imprimerie Nationale.

Résultats statistiques du recensement général de la population du 8 mars 1936, (1938), Imprimerie Nationale,.

Ter-Minassian, Anahide, « Les Arméniens en France », Dossiers d'archéologie, $\mathrm{n}^{\circ}$ 177, décembre 1992, (pp. 132-135).

Weber, Eugen, (1995), La France des années 30. Tourments et perplexités, Paris, Fayard.

Zalc, Claire, (2003), « De la liberté du commerce pour tous à carte de commerçant étranger », in Bruno, Anne-Sophie et Zalc, Claire (dir.) Petites entreprises et petits indépendants étrangers en France (XIX et XX ${ }^{e}$ siècle), Paris : Éditions Publibook, (pp. 29-48).

\section{ANNEXES}

\section{Archives exploitées}

\section{Archives de Paris}




\begin{tabular}{|l|l|}
\hline Cote & Contenu \\
\hline D.33U3 & Registre du commerce du département de la Seine \\
\hline 2MILN 1926 & Listes nominatives du recensement de 1926 de la ville de Paris \\
\hline 2MILN 1931 & Listes nominatives du recensement de 1931 de la ville de Paris \\
\hline 2MILN 1936 & Listes nominatives du recensement de 1936 de la ville de Paris \\
\hline
\end{tabular}

Archives de la préfecture de Police du département de la Seine

\begin{tabular}{|l|l|}
\hline Cote & Contenu \\
\hline BA 1681 & Colonies étrangères \\
\hline BA 2146 & Arménie colonie arménienne à Paris \\
\hline BA 2327 & Arménie colonie arménienne à Paris \\
\hline BA 2328 & Arménie colonie arménienne à Paris \\
\hline BA 2329 & Arménie colonie arménienne à Paris \\
\hline BA 2273 & Communauté juive, dossiers divers associations \\
\hline BA 2314 & Communauté juive, amicales, associations \\
\hline BA 2315 & Communauté juive, amicales, associations \\
\hline BA 1811 & Communauté juive, prolétariat juif français \\
\hline
\end{tabular}

\section{RÉSUMÉS}

Les points communs et différences des stratégies d'intégration des Arméniens, Grecs et Juifs, originaires de Grèce et de Turquie, à Paris, 1920-36.

Cet article est un résumé du livre « Les Arméniens les Grecs et les Juifs originaires de Grèce et de Turquie, à Paris entre 1920 et 1936 » paru aux éditions Isis en décembre 2010. Cinq groupes d'immigrés ont été définis en croisant l'origine communautaire et géographique : les Arméniens de Turquie, les Grecs de Grèce, les Grecs de Turquie, les Juifs de Grèce et les Juifs de Turquie. L'article analyse d'abord les causes politiques et économiques de cette immigration hétérogène, en précise les flux et l'implantation à Paris, puis il décrit le processus communautaire d'intégration de ces immigrés dans la société française. Il montre ensuite que la politique d'immigration des autorités françaises et la crise économique limitent l'accès de ces immigrés à l'emploi salarié. L'activité indépendante se développe au sein de cette immigration comme une stratégie alternative d'intégration professionnelle. Il existe de nombreux points communs à l'activité indépendante dans les différents groupes, notamment l'augmentation continue du taux 
d'indépendance professionnelle pendant la période ainsi que la similitude des moteurs et le processus de développement de l'activité indépendante. Mais il y aussi des différences sensibles entre les groupes quant au taux d'indépendance ou d'activité salariée et les activités professionnelles.

This paper gives the main lines of the book "Armenians, Greeks and Jews born in Greece and Turkey and living in Paris, 1920-1936", published in December 2010 by the Isis editions. According to the cultural and geographical origin, five immigrant groups were distinguished: Armenians from Turkey, Greeks from Greece and Greeks from Turkey, Jews from Greece and Jews from Turkey. The paper deals first with the political and economical reasons of this heterogeneous immigration, details the main streams and their implantation in Paris, and then, describes the process of the community integration in the French society. After that, it shows how the immigration policy of the French authorities and the economical crisis restrains the access of these immigrants to the wage-earning work. Self- employed activities were developed among these immigration as alternative strategy for professional integration. Many points common to the different groups can be found as concerns the self-employed activities, as the continuous augmentation of the professional self-employment rate and the similar driving forces and development process. Nevertheless we found real differences between the groups as to selfemployment rate or the wage-earning activities and the different professional activities.

\section{INDEX}

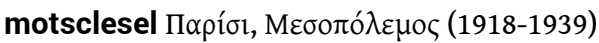

motsclesmk ПАРИЗ

motsclestr Savaşlar arasındaki (1918-1939)

Mots-clés : Arméniens à Paris, immigrés -- Paris, étrangers à Paris, Grecs à Paris, Juifs à Paris

Thèmes : Histoire

Index chronologique : entre-deux-guerres (1918-1939)

Index géographique : Paris

Keywords : Greeks in Paris, Armenians in Paris, integration in France, Interwar period

(1918-1939), History

\section{AUTEUR}

\section{MICHEL GARIN}

Docteur, Chercheur associé CREE-CEB EA 4513 